\title{
Mesenchymal stem cell-derived exosomes as a new therapeutic strategy for liver diseases
}

\author{
Guohua Lou ${ }^{1,2}$, Zhi Chen ${ }^{1,2}$, Min Zheng ${ }^{1,2}$ and Yanning Liu ${ }^{1,2}$
}

The administration of mesenchymal stem cells (MSCs) as a therapy for liver disease holds great promise. MSCs can differentiate into hepatocytes, reduce liver inflammation, promote hepatic regeneration and secrete protective cytokines. However, the risks of iatrogenic tumor formation, cellular rejection and infusional toxicity in MSC transplantation remain unresolved. Accumulating evidence now suggests that a novel cell-free therapy, MSC-secreted exosomes, might constitute a compelling alternative because of their advantages over the corresponding MSCs. They are smaller and less complex than their parent cells and, thus, easier to produce and store, they are devoid of viable cells, and they present no risk of tumor formation. Moreover, they are less immunogenic than their parent cells because of their lower content in membrane-bound proteins. This paper reviews the biogenesis of MSC exosomes and their physiological functions, and highlights the specific biochemical potential of MSC-derived exosomes in restoring tissue homeostasis. In addition, we summarize the recent advances in the role of exosomes in MSC therapy for various liver diseases, including liver fibrosis, acute liver injury and hepatocellular carcinoma. This paper also discusses the potential challenges and strategies in the use of exosome-based therapies for liver disease in the future. Experimental \& Molecular Medicine (2017) 49, e346; doi:10.1038/emm.2017.63; published online 16 June 2017

\section{INTRODUCTION}

Mesenchymal stem cell (MSC)-based therapy has emerged as a promising strategy for treating liver diseases via tissue repair and immune regulation. ${ }^{1-3}$ However, the use of MSCs has some drawbacks, such as the need of a consistent supply of cells with stable phenotype, high costs and time delays for the generation and handling of these cells. In addition, issues related to ectopic tissue formation, infusional toxicity caused by cells lodged in the pulmonary microvasculature, and cellular rejection or unwanted engraftment, have been reported. ${ }^{4}$ Studies have shown that MSCs can achieve a therapeutic effect in vivo via paracrine action ${ }^{5,6}$ and direct differentiation. Subsequent studies indicated that MSC-secreted extracellular vesicles (EVs), including microvesicles (MVs; 0.1-1 $\mathrm{mm}$ in diameter) and exosomes (40-100 nm in diameter), ${ }^{7,8}$ may contribute to the therapeutic potency of MSCs by mediating cell-cell micro-communication and transporting paracrine factors during angiogenesis, tissue regeneration and immune regulation. ${ }^{9-12}$

The administration of MSC-derived exosomes has yielded beneficial effects in a variety of animal models of liver disease, including drug-induced acute liver injury, liver fibrosis and hepatocellular carcinoma (HCC). ${ }^{13-16}$ Exosomes have advantages over the corresponding MSCs: they are smaller and less complex than cells, so they are easier to produce and store, and have the potential to avoid some of the regulatory issues that face MSCs. ${ }^{17}$ Therefore, MSC-derived exosomes may represent an ideal therapeutic tool for liver diseases in the near future.

\section{FUNCTIONS OF EXOSOMES}

Exosomes are nano-sized EVs that possess remarkable physiological properties and originate via the inward budding of the membrane of late endosomes called multivesicular bodies (MVBs). Upon the fusion of MVBs with the plasma membrane, exosomes are released into the extracellular milieu and can be either taken up by target cells residing in the microenvironment or carried to distant sites via biological fluids. ${ }^{18}$ Exosomes have a narrow diameter range of $40-100 \mathrm{~nm}$ and a density of $1.13-1.19 \mathrm{~g} \mathrm{ml}^{-1}$ in sucrose solution. They can be sedimented by centrifugation at $100000 \mathrm{~g} .{ }^{19}$ Exosome membranes are enriched in cholesterol, sphingomyelin, ceramide and lipid raft proteins. ${ }^{20}$ Exosomes are reported to contain both proteins and RNAs. ${ }^{21}$ Most exosomes have an evolutionary conserved set of proteins including tetraspanins

\footnotetext{
${ }^{1}$ State Key Laboratory for Diagnosis and Treatment of Infectious Diseases, The First Affiliated Hospital, College of Medicine, Zhejiang University, Hangzhou, China and ${ }^{2}$ Collaborative Innovation Center for Diagnosis and Treatment of Infectious Diseases, Hangzhou, China

Correspondence: Dr Y Liu, State Key Laboratory for Diagnosis and Treatment of Infectious Diseases, The First Affiliated Hospital, Zhejiang University, 79\# Qingchun Road, 6A-17, Hangzhou 310003, China.

E-mail: liuyanning@zju.edu.cn

Received 31 August 2016; revised 3 January 2017; accepted 12 January 2017
} 
(CD81, CD63 and CD9), heat-shock proteins (HSP60, HSP70 and HSP90), ALIX and tumor susceptibility gene 101 (TSG101); however, they also have unique tissue/cell type-specific proteins that reflect their cellular source. ${ }^{22}$

\section{General physiological functions of exosomes}

Exosomes were once thought to be 'trash bags' for cells to discard unwanted proteins. However, over the past decade, they have emerged as an important intercellular communication vehicle for modulating or mediating a variety of cellular processes. Exosome release is currently considered an integral mechanism of cell-to-cell communication that may affect both neighboring cells and distant parts of the body.

In the immune system, exosomes have been demonstrated to have a key role in mediating both adaptive and innate immune responses, thereby participating in antigen presentation by spreading antigens in association with MHC-II and MHC-I molecules. For example, B-lymphocyte- and dendritic cell (DC)-derived exosomes stimulate $\mathrm{T}$ cells and initiate host immune responses. ${ }^{23,24}$ When pulsed with tumor peptides, DC-derived exosomes prime cytotoxic $\mathrm{T}$ cells and suppress tumor growth in mice. ${ }^{25}$

Interestingly, virally or bacterially infected cells use exosomes as vehicles to disseminate disease. ${ }^{26,27}$ The exosomes that are secreted from scrapie-infected epithelial and neuroglial cells, which contain infectious prion protein (PrP) scrapie (PrPsc), may contribute to intercellular membrane exchange and the spread of prions throughout the organism. ${ }^{28}$ Exosomes derived from cells infected by human immunodeficiency virus type $1,{ }^{29}$ Epstein-Barr virus, ${ }^{30}$ Herpes simplex virus- $1^{31}$ and hepatitis C virus $^{32}$ circulate in the bloodstream, attack and enter their target cells. Viruses that are delivered by exosomes are released and replicate within them, giving rise to a systematic distribution of viral agents that influence the host immune cells. ${ }^{33}$ Similar to virally infected cells, bacterially infected cells such as Mycobacterium tuberculosis-, Mycobacterium bovis- or Mycobacterium avium-infected macrophages release exosomes that contain pathogen-derived antigens, ${ }^{34}$ which have an important role in immune surveillance during intracellular bacterial infections.

Cancer cells also utilize exosomes in tumor progression and metastasis. Highly metastatic melanoma cell-derived exosomes can promote epithelial-mesenchymal transition in primary melanocytes. ${ }^{35}$ Glioblastoma multiforme cell-derived hypoxic exosomes can secrete and transfer potent growth factors into vascular cells to promote tumor vascularization during tumor development. ${ }^{36}$ Tumors such as melanoma and colorectal carcinoma also secrete exosomes to induce immunosuppressive myeloid cells and possibly enable tumors to evade the immune system. ${ }^{37}$ The nucleic acid content of exosomes also appears to be important in tumor metastasis; for instance, the PTEN-targeting microRNAs, which are transferred by astrocyte-derived exosomes, enhance brain metastasis outgrowth. ${ }^{38}$

Recently, exosomes have also been implicated in degenerative diseases. In Parkinson's and Alzheimer's patients, neuron- secreted exosomes containing $\alpha$-synuclein ${ }^{39}$ and amyloid- $\beta$ peptide $(\mathrm{A} \beta),{ }^{40}$ respectively, have been reported. These exosomes can contribute to the nucleation or physical dissemination of the $\alpha$-synuclein and $A \beta$ aggregates that characterize the two diseases. Another study showed that exosomes from interleukin-1 $\beta$ (IL-1 $\beta$ )-stimulated human synovial fibroblasts could induce osteoarthritis-like changes in both in vitro and ex vivo models: this might represent a novel mechanism involving osteoarthritis-affected joints via pathogenic signal communication. ${ }^{41}$

Moreover, exosomes are emerging as mediators in tissue regeneration. During injury, cardiomyocyte progenitor cells secrete exosomes to stimulate the migration of endothelial cells and promote cardiac regenerative activity. ${ }^{42}$ Exosomes have also been shown to be involved in the regeneration of peripheral nerves and in the repair of neuronal injuries. ${ }^{43}$ Furthermore, their ability to cross the blood-brain barrier has prompted extensive investigations to use them as delivery vehicles to treat neurological disorders. ${ }^{44}$

\section{Functions of MSC-derived exosomes}

The physiological function of MSC-derived exosomes has not been defined. They may act as an intercellular communication vehicle for modulating or mediating cellular processes, similar to the exosomes derived from cell types. MSC-derived exosomes may interact with multiple cell types within adjacent and remote areas to elicit appropriate cellular responses; they affect the stromal support functions of MSCs through the maintenance of a dynamic and homeostatic tissue microenvironment. ${ }^{45}$

Similar to exosomes in general, MSC-derived exosomes carry complex cargo, including nucleic acids, proteins and lipids. Through mass spectrometry, antibody array and microarray analysis, $>850$ unique gene products and $>150$ miRNAs have been identified in the cargo of MSC-derived exosomes. ${ }^{46,47}$ These exosomal proteins and miRNAs are functionally complex, and are implicated in many diverse biochemical and cellular processes, such as communication, immune regulation, bioenergetics, tissue repair and regeneration and metabolism. Thus, MSC-derived exosomes display the potential to elicit diverse cellular responses and interact with various cell types.

MSC-derived exosomes have a key role in mediating the capacity of MSCs to function as stromal support cells to maintain homeostasis within the tissue and respond to external stimuli. This role is particularly important when the homeostasis of the tissue microenvironment is disrupted by disease or injury; this, in turn, compromises normal tissue function. MSC-derived exosomes are highly enriched in biologically active molecules, such as proteins and RNAs, and are therefore well equipped for this role. ${ }^{48}$ Many of the proteins found in exosomes are enzymes, with activities that are catalytic rather than stoichiometric and are dictated by their microenvironment (for example, substrate concentration or $\mathrm{pH}$ ). Therefore, the enzyme-centric feature of exosomes may alleviate the risk of over- or under-dosing if they are used as therapeutical agents. ${ }^{49} \mathrm{~A}$ significant clustering of glycolytic enzymes has been 
found in MSC exosomes; if they are given as therapy, these enzymes from MSC exosomes can ameliorate the glycolytic deficit and potentially increase glycolytic flux and ATP production in the reperfused myocardium. ${ }^{50}$

Moreover, exosomes are the ideal vehicles to protect and deliver molecules to the appropriate targets. By encapsulating molecules within their membranes, exosomes can protect enzymes or RNAs against degradation and facilitate their intracellular uptake via the cellular endocytosis of exosomes. ${ }^{51,52}$ The uptake of exosomes has been reported to be modified by microenvironmental acidity, ${ }^{53}$ and tissue injury is often characterized by tissue acidosis. ${ }^{54}$ Exosomes would be preferentially taken up by cells in injured tissues. Furthermore, exosomes are nanometer-sized particles that can be easily transferred through blood and other biological fluids. Thus, MSC-derived exosomes can mediate cell communication in both adjacent and remote areas via paracrine and endocrine signaling. The capacity of MSC-derived exosomes to restore and maintain the homeostasis of the tissue microenvironment would depend on the biochemical potential of their protein and RNA cargo.

\section{THERAPEUTIC PROPERTIES OF MSC-DERIVED EXOSOMES ON LIVER DISEASE}

The unique ability of MSCs to self-renew and their multipotentiality have made them a promising cell-based strategy for treating liver diseases. Recent studies have shown that MSC-derived exosomes are as potent as parent stem cells in the regeneration of various organ injury models, primarily through the transfer of their cargo to the recipient cells, which are consequently modified in their function and/or phenotype. ${ }^{55,56}$ As shown in Table 1, multiple recent studies have presented preclinical data addressing the reparative and regenerative properties of MSC-derived exosomes in liver diseases. ${ }^{13-16,57}$

\section{MSC-derived exosomes for liver fibrosis}

The application of MSCs in animal models of liver fibrosis/ cirrhosis and, eventually, in patients ameliorates the progress of the disease. ${ }^{58}$ Similar results were obtained when MSCconditioned media (MSC-CM) were applied instead, ${ }^{58,59}$ which suggests that MSCs might achieve a therapeutic effect in vivo via their secreted EVs, such as exosomes.

Several studies have focused on the therapeutic effects of MSC-derived exosomes in a mouse model of liver fibrosis. Using a carbon tetrachloride $\left(\mathrm{CCl}_{4}\right)$-induced liver injury model in Kunming mice, $\mathrm{Li}$ et al. found that the exosomes derived from human umbilical cord MSCs ameliorate liver fibrosis by inhibiting both the epithelial-mesenchymal transition of hepatocytes and collagen production. Exosomes were found to significantly restore the serum aspartate aminotransferase activity and inactivate the TGF- $\beta 1 /$ Smad signaling pathway by decreasing collagen type I/III and TGF- $\beta 1$ and the phosphorylation of Smad2. ${ }^{14}$ Another study showed that chorionic platederived MSCs could release exosomes containing miR-125b, mediate miR-125b transfer between MSCs and target cells, such as Hedgehog (Hh)-responsive hepatic stellate cells
(HSCs), and thus alleviate hepatic fibrosis in $\mathrm{CCl}_{4}$-treated Sprague-Dawley rats by impeding the activation of $\mathrm{Hh}$ signaling via the inhibition of Smo expression. ${ }^{15}$ Our unpublished data showed that the exosomes produced by adipose tissue-derived MSCs (AD-MSC-122) expressing miR-122 were more effective than were those expressing scramble miRNA or naive exosomes in reducing the proliferation and activation of the human HSC cell line LX2 or primary HSCs from C57BL/6 mice. AD-MSC-122-derived exosomes could transfer miR-122 into HSCs cells and then regulate the expression of miR-122-target genes, such as P4HA1 and IGF1R, which have been shown to be involved in the proliferation and collagen maturation of HSCs. ${ }^{60,61}$ These data indicate that miR-122-modification may improve the therapeutic efficacy of AD-MSCs via exosome-mediated miR-122 delivery, thereby representing a new strategy for treating liver fibrosis.

\section{MSC-derived exosomes for acute liver injury}

The therapeutic effects of MSC MVs have been reported in several experimental models of acute kidney, cardiac, neural and lung injury. In acute kidney injury models induced by cisplatin, ${ }^{62}$ glycerol, ${ }^{63}$ ischemia-reperfusion (I/R), ${ }^{64}$ nephrectomy ${ }^{65}$ and drug toxicity (gentamicin), ${ }^{66}$ MSC exosomes or MVs can improve renal function and reduce the extent of kidney damage. A single administration of MSC exosomes immediately following cisplatin- or I/R-induced acute kidney injury alleviates inflammation, mitigates renal cell apoptosis, and enhances the proliferation of renal epithelial cells. ${ }^{10,62}$ In myocardial $\mathrm{I} / \mathrm{R}$ injury, the administration of purified MSC exosomes before reperfusion significantly reduces the infarct size and improves the left ventricular function. ${ }^{67,68}$ Further studies have shown that MSC-derived exosomes exert protective effects against myocardial $\mathrm{I} / \mathrm{R}$ injury possibly through several mechanisms, such as anti-apoptosis, cardiac regeneration, anti-cardiac remodeling, anti-inflammatory effects, neovascularization and anti-vascular remodeling. ${ }^{69,70}$

However, to date, only a few groups have studied the therapeutic effects of MSC exosomes in acute liver injury. ${ }^{13}$ Tan et al. found that HuES9.E1 MSC-derived exosomes elicit hepatoprotective effects both in in vitro models of acetaminophen or $\mathrm{H}_{2} \mathrm{O}_{2}$-induced hepatocyte injury and in a C57BL/6 mouse model of $\mathrm{CCl}_{4}$-induced acute liver injury, primarily through an increase in hepatocyte proliferation, as demonstrated by elevated proliferating cell nuclear antigen and high cell viability. The increased survival rate is associated with the upregulation of genes involved in the priming phase liver regeneration, which subsequently lead to high expression of proliferation proteins (proliferating cell nuclear antigen and Cyclin D1), the anti-apoptotic gene Bcl-xL and the signal transducer and activator of transcription 3 (STAT3). However, the therapeutic effect of MSC-derived exosomes does not occur via the modulation of oxidative stress during hepatic injury. ${ }^{13}$ At the CSH-AASLD \& CSH-EASL Joint Scientific Symposiums meeting on Hepatology (Beijing, 2016), we presented our study indicating that transplantation of exosomes released 
Table 1 Application of MSC-derived exsomes in liver diseases

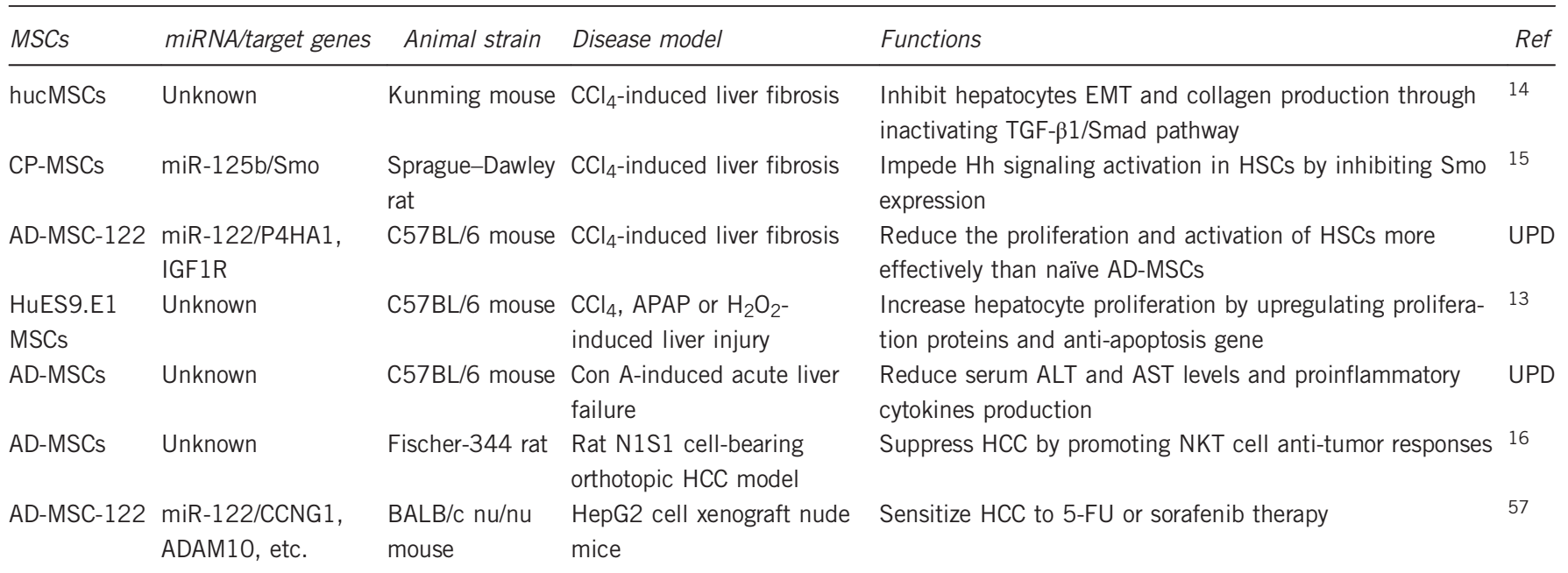

Abbreviations: AD-MSCs, adipose tissue-derived MSCs; AD-MSC-122, miR-122-expressed AD-MSCs; ALT, alanine aminotransferase; AST, aspartate aminotransferase; CP-MSCs, chorionic plate-derived MSCs; HuES9.E1 MSCs, hESC-derived HuES9.E1 MSCs; hucMSCs, human umbilical cord MSCs; UPD, our unpublished data; 5-Fu, 5-fluorouracil.

from AD-MSC can significantly reduce the elevated serum levels of alanine aminotransferase and aspartate aminotransferase, liver inflammation and necrosis in concanavalin A (Con A)-induced hepatitis in C57BL/6 mice as well as the serum levels of proinflammatory cytokines, including tumor necrosis factor- $\alpha$ (TNF- $\alpha$ ), interferon- $\gamma$ (IFN- $\gamma$ ), IL-6, IL-18 and IL-1 $\beta$, and the inflammasome activation in mouse liver.

MSCs offer an opportunity to treat acute liver injury induced by lipopolysaccharide (LPS), ${ }^{71}$ thioacetamide, ${ }^{72} \mathrm{I} / \mathrm{R},{ }^{73}$ and radiation, ${ }^{74}$ and $\mathrm{MSC}-\mathrm{CM}$ has been shown to exert a protective effect on these models. ${ }^{75,76}$ Thus, we have reason to believe that the transplantation of MSC-derived exosomes may be a novel therapeutic approach for treating various types of acute liver injury.

\section{MSC-derived exosomes for HCC}

MSCs have recently gained much attention for their application to tumor therapy because MSCs can mobilize from the bone marrow or other tissues to the tumor microenvironment. ${ }^{77,78}$ Several studies have shown that MSCs can either promote or inhibit tumor progression in different tumor models. ${ }^{79-82}$ However, the mechanisms by which MSCs control tumor cells remain unclear.

MSC-secreted paracrine factors, which are delivered by EVs, have been shown to mediate their effects on tumor progression. $^{83}$ For example, exosomes that are released from multiple myeloma patient bone marrow (BM)-derived MSCs promote multiple myeloma tumor growth in SCID-beige mice. ${ }^{84}$ MVs derived from human Wharton's jelly MSCs promote renal cancer cell growth and aggressiveness in BALB/c nu/nu mice. ${ }^{85}$ BM-MSC-derived exosomes promote gastric or colon tumor growth in $\mathrm{BALB} / \mathrm{c} \mathrm{nu} / \mathrm{nu}$ mice by enhancing the expression of vascular endothelial growth factor in tumor cells, ${ }^{86}$ and they facilitate nasopharyngeal carcinoma progression and migration in non-obese diabetic/severe combined immunodeficient
(NOD/SCID) mice by activating the FGF19-FGFR4-dependent ERK signaling cascade and epithelial-mesenchymal transition. ${ }^{87}$ However, exosomes also exhibit anti-tumor effects. Bruno et al. ${ }^{88}$ found that exosomes from human BM-MSCs inhibit the growth and survival of three different human tumor cell lines, and similar results were observed in NOD/SCID mouse models. In another study, mouse BM-MSC-derived exosomes were found to suppress tumor progression and angiogenesis in the mouse breast cancer cell line 4T1 by downregulating the expression of vascular endothelial growth factor in vitro and in vivo via shuttling miR-16, which is a known effector of vascular endothelial growth factor that is enriched in MSC-derived exosomes. ${ }^{89}$ A different study showed that exosomes derived from menstrual stem cells suppress the secretion of pro-angiogenic factors in prostate tumor cell line PC3 in a reactive oxygen species-dependent manner and inhibit angiogenesis of prostate tumor in PC3-bearing NOD SCID gamma mice. ${ }^{90}$ The different effects of MSC-derived exosomes on tumor growth may be due to the heterogeneity of MSCs. MSCs from different donors or tissue sources may release exosomes containing cell-specific molecules to promote or prevent tumor development. In addition, the different tumor types and in vivo tumor models examined and the variation in the way and time exosomes are administered may also influence the effect of exosomes on tumor progression. ${ }^{91}$

To date, only a few studies have shown the effect of MSC exosomes on HCC. MVs derived from BM-MSCs inhibit cell cycle progression and induce apoptosis in HepG2 cells. The intra-tumor administration of MVs in established tumors generated by a subcutaneous injection of HepG2 cells in SCID mice significantly inhibits tumor growth. ${ }^{88} \mathrm{~A}$ recent study showed that AD-MSC-derived exosomes in rat N1S1 cells, which is an orthotopic HCC model, can promote NKT cell anti-tumor responses in rats, thereby facilitating HCC suppression, low-grade tumor differentiation and an increase of 
the early apparent diffusion coefficient, which is a measure of the magnitude of water diffusion within the tissue and can be used as an early biomarker of treatment response. ${ }^{16}$

In addition to their role in modulating tumor development, MSCs-derived exosomes influence tumor chemosensitivity. Exosomes from human umbilical cord MSCs significantly induce the resistance of gastric cancer cells to 5-fluorouracil in a BALB/c nu/nu mice subcutaneous xenograft tumor model by antagonizing 5 -fluorouracil-induced apoptosis by enhancing the expression of multidrug resistance-associated proteins. ${ }^{92} \mathrm{In}$ another study, exosomes from anti-miR-9-transfected BM-MSCs delivered anti-miR-9 into temozolomide-resistant glioblastoma multiforme cells and reversed their chemoresistance by affecting the expression of the multidrug transporter P-glycoprotein. ${ }^{93}$

In one of our previous studies, we found that naïve AD-MSC-derived exosomes have no effect on tumor growth and chemosensitivity in HepG2 cell xenografts in BALB/c $\mathrm{nu} / \mathrm{nu}$ mice. Because miR-122 has an essential role in increasing the chemosensitivity of HCC cells by targeting CCNG1, ADAM10 and IGF1R, we modified AD-MSCs with miR-122. Exosomes from miR-122-modified AD-MSC (122-Exo) could mediate miR-122 transfer between AD-MSCs and HCC cells, thereby enhancing cell sensitivity to chemotherapeutic agents by regulating miR-122-target gene expression in HCC cells. Moreover, an intra-tumor injection of 122-Exo significantly sensitizes HCC to sorafenib therapy in vivo. ${ }^{57}$ The different anti-cancer effects of AD-MSC-derived exosomes on HCC between our study and the study performed by Ko et al. in a rat model may be due to differences in the tumor model of HCC investigated. In a previous study, Ko et al. ${ }^{16}$ used N1S1 rat HCC cells to obtain an orthotopic HCC model in Fischer-344 (F344) rats, which have a normal immune system. They found that AD-MSC-derived exosomes promote NKT cell anti-tumor responses in rats, thereby facilitating HCC suppression. This finding suggests that the anti-cancer effect of AD-MSC-derived exosomes is mainly by promoting anti-cancer immunity. In our study, we used HepG2 human HCC cells to construct a subcutaneous xenograft model in nude mice in which the immune-mediated anti-cancer effect of AD-MSC-derived exosomes could not be observed.

The use of MSC-derived exosomes in cancer therapy must be conducted with caution because their role in tumor growth remains unclear. A better understanding of the mechanisms involved in the regulation of MSC-derived exosomes is important to determine their true role in cancer progression and guide researchers in the development of important therapeutic agents through their specific modification.

\section{MSC-derived exosomes for drug delivery}

Advances in biomedical research have generated an increasing number of potential targets for treating liver disease or delaying disease progression. Unfortunately, many of these targets are undruggable because they are intracellular, present in many cell types, poorly soluble or rapidly inactivated. Although pharmaceutical drug vehicles (for example, synthetic lipid and nanoparticles), have successfully circumvented many of these problems, exosomes, as a mimic of 'nature's delivery systems,' are highly attractive as a potential alternative to deliver drugs to target cells via membrane fusion or endocytosis. Given the problems associated with many of the current nanoparticulate delivery systems, exosomes can avoid phagocytosis or degradation by macrophages and also circulate for extended time periods within the body because of nature's own cellular product. Unlike typical nanoparticulate systems such as liposomes or polymeric nanoparticles, exosomes can potentially avoid the endosomal pathway and lysosomal degradation, and deliver cargoes directly into the cytoplasm. By avoiding the endosomal pathway, the transfection efficiency for molecules such as siRNA can be enhanced..$^{94}$ Moreover, exosomes have other features of an ideal drug delivery vehicle. First, the presence of proteins and RNAs in exosomes indicates that they can be loaded with such biological materials. ${ }^{95}$ Second, exosomes can penetrate the blood-brain barrier, ${ }^{96}$ which has proven to be highly impenetrable to many drugs. Third, exosomes are naturally stable and have inherent targeting properties depending on their composition..$^{97,98}$ Finally, exosomes are amenable to membrane modifications that enhance cell type-specific targeting. ${ }^{99,100}$

Among the cell types that are known to produce exosomes, MSC are the ideal candidate for the mass production of exosomes for drug delivery. At present, MSC-derived exosomes have been used as drug delivery vehicles in tumor therapy and regenerative medicine in some studies. ${ }^{101,102}$ Upon in vitro exposure to high concentrations of paclitaxel (PTX), BM-MSCs can package and deliver this active drug through their exosomes. The PTX-loaded exosomes acquire strong anti-tumor effects on human pancreatic adenocarcinoma (CFPAC-1), in vitro. ${ }^{103}$ Exosomes from BM-MSCs overexpressing miR-146b or miR-143 can significantly reduce the growth of glioma xenografts in a Fischer rat model or the migration of osteosarcoma cells in vitro, through delivery of these antitumor miRNAs into the target tissue or cells, respectively. ${ }^{104,105}$ BM-MSCs loaded with anti-miR-9 can transfer this anti-miR into co-cultured-glioblastoma cells via secreted exosomes, thereby conferring temozolomide chemosensitivity. ${ }^{93}$

In addition to their use in tumor therapy or chemotherapy, MSC exosome-shuttled therapeutic materials have been used in regenerative medicine. CXCR4-enriched exosomes released from CXCR4-overexpressing rat BM-MSCs can protect cardiomyocytes from ischemic injury both in vitro and in a rat model of myocardial infarction. Upregulation of the Akt signaling pathway contributes to these beneficial effects, suggesting that CXCR4-enriched exosomes may serve as an additional therapeutic strategy to promote cell survival and angiogenesis in ischemic hearts following myocardial infarction. ${ }^{106}$

To date, there are few studies on the use of MSC-derived exosomes for drug delivery in liver diseases. Because specifically modified MSCs and their conditioned media show better therapeutic efficacy on liver diseases ${ }^{1,107}$, and because exosomes that contain therapeutic molecules can be mass produced from 


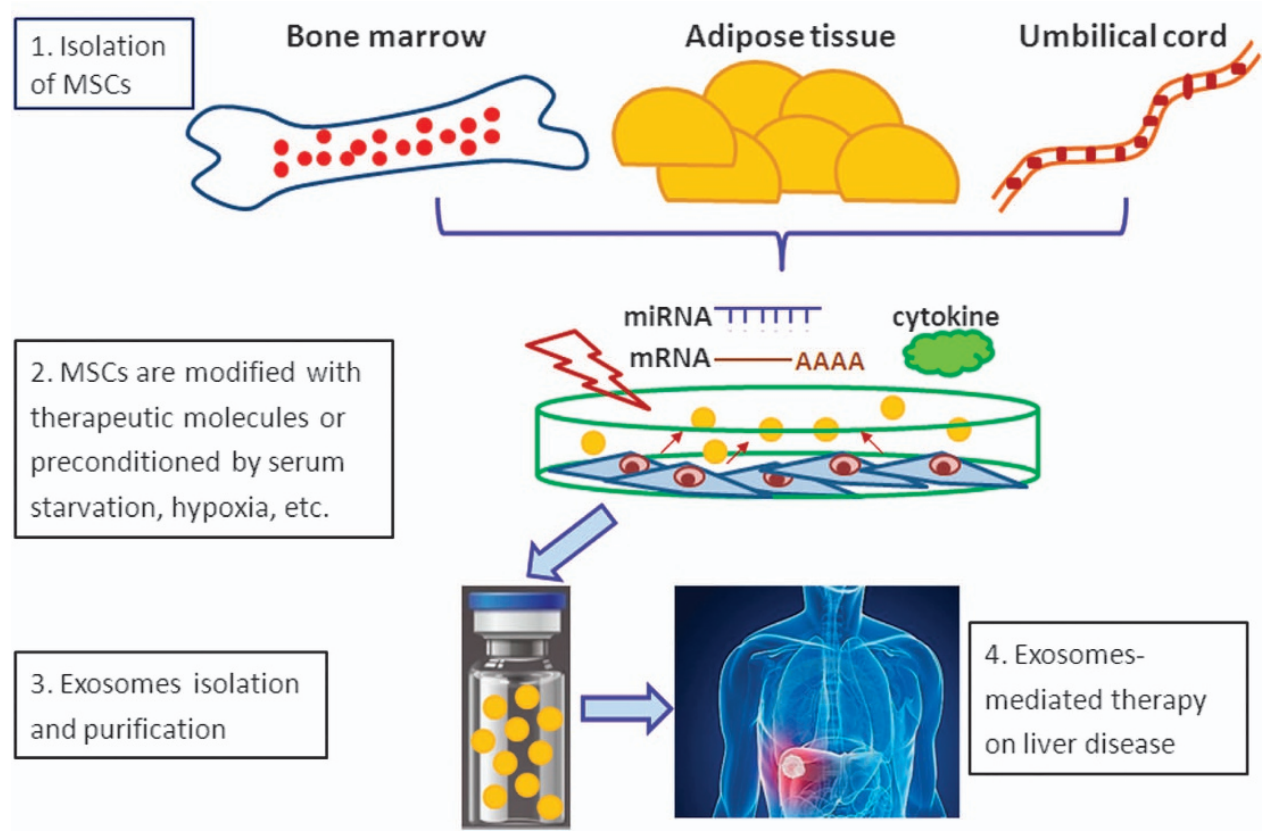

Figure 1 MSC-derived exosomes represent an attractive therapeutic approach for treating liver diseases. Step 1: MSCs are isolated from bone marrow, adipose tissue and umbilical cord. Step 2: MSCs are modified with therapeutic molecules (i.e., mRNA, miRNAs and cytokines) or preconditioned by serum starvation, hypoxia and pharmacological and physical stimulation. Step 3: Exosomes loading therapeutic molecules are isolated from MSC-conditioned media, purified, characterized and quantified. Step 4: Administration of exosomes via veins (i.e., portal vein) to treat liver diseases, including acute liver injury, liver fibrosis and HCC. MSCs, mesenchymal stem cells; HCC, hepatocellular carcinoma.

MSCs, exosome-mediated drug delivery potentially engenders a novel approach for treating liver diseases such as hepatitis, liver fibrosis and HCC.

\section{CONCLUSIONS AND PROSPECTS}

As stromal support cells, MSCs target housekeeping processes through their secreted exosomes to restore liver homeostasis and enable hepatocytes to recover, repair and regenerate. This observation provides the rationale for the therapeutic efficacy of MSCs and their secreted exosomes in a variety of liver diseases. Furthermore, by maintaining the therapeutic advantages of MSCs without the risk of iatrogenic tumor formation or of pulmonary embolisms due to intravenous administration, MSC-derived exosomes represent a highly attractive therapeutic approach for treating inflammatory liver diseases and HCC. Such exosomes also can be used as an adjuvant to support and complement other therapeutic modalities (Figure 1).

However, questions about the characterization, potency and quantification of MSC exosomes must be addressed before their clinical use. On the basis of preclinical studies, the amount of MSC exosomes needed to generate an equivalent effect as MSCs in tissue injury is roughly higher. ${ }^{7}$ Although MSCs are relatively easy to expand in vitro, their growth in culture is finite. Both new batches of MSCs, through immortalization and expansion by bioreactors, and more effective techniques for large-scale exosome production need to be developed. Moreover, there is still no gold standard to characterize MSC exosomes. Because MSCs are heterogeneous, the exosomes isolated from them are also heterogeneous, and this heterogeneity may cause different effects on their target cells. ${ }^{84,108}$ In addition, the methods used to precondition MSC in stimulating exosome release, such as serum starvation, hypoxia and inflammation, change the surface and intracellular content of exosomes. Thus, to significantly advance exosomemediated therapy in clinical trials, effective methods that maintain the homogeneity of MSC-derived exosomes, involve robust and standardized characterization of MSC-derived exosomes, and include standardized safety data from preclinical animal models need to be developed.

\section{CONFLICT OF INTEREST}

The authors declare no conflict of interest.

\section{ACKNOWLEDGEMENTS}

This work was supported by the National Natural Science Fund (81000730 and 81201782), and the Key R\&D projects of the Zhejiang province (2015C03045).

1 Kim MD, Kim SS, Cha HY, Jang SH, Chang DY, Kim W et al. Therapeutic effect of hepatocyte growth factor-secreting mesenchymal stem cells in a rat model of liver fibrosis. Exp Mol Med 2014; 46: e110.

2 Christ B, Brückner S, Winkler S. The therapeutic promise of mesenchymal stem cells for liver restoration. Trends Mol Med 2015; 21: 673-686.

3 Volarevic V, Nurkovic J, Arsenijevic N, Stojkovic M. Concise review: therapeutic potential of mesenchymal stem cells for the treatment of acute liver failure and cirrhosis. Stem Cells 2014; 32: 2818-2823. 
4 Urbanelli L, Buratta S, Sagini K, Ferrara G, Lanni M, Emiliani C. Exosomebased strategies for diagnosis and therapy. Recent Pat CNS Drug Discov 2015; 10: 10-27.

5 Gnecchi M, Danieli P, Malpasso G, Ciuffreda MC. Paracrine mechanisms of mesenchymal stem cells in tissue repair. Methods Mol Biol 2016; 1416: 123-146.

6 Gnecchi M, He H, Liang OD, Melo LG, Morello F, Mu H et al. Paracrine action accounts for marked protection of ischemic heart by Akt-modified mesenchymal stem cells. Nat Med 2005; 11: 367-368.

7 Phinney DG, Di Giuseppe M, Njah J, Sala E, Shiva S St, Croix CM et al. Mesenchymal stem cells use extracellular vesicles to outsource mitophagy and shuttle microRNAs. Nat Commun 2015; 6: 8472.

8 Wen D, Peng Y, Liu D, Weizmann Y, Mahato RI. Mesenchymal stem cell and derived exosome as small RNA carrier and Immunomodulator to improve islet transplantation. J Control Release 2016; 238: 166-175.

9 Arslan F, Lai RC, Smeets MB, Akeroyd L, Choo A, Aguor EN et al. Mesenchymal stem cell-derived exosomes increase ATP levels, decrease oxidative stress and activate PI3K/Akt pathway to enhance myocardial viability and prevent adverse remodeling after myocardial ischemia/ reperfusion injury. Stem Cell Res 2013; 10: 301-312.

10 Lin KC, Yip HK, Shao PL, Wu SC, Chen KH, Chen YT et al. Combination of adipose-derived mesenchymal stem cells (ADMSC) and ADMSC-derived exosomes for protecting kidney from acute ischemia-reperfusion injury. Int J Cardiol 2016; 216: 173-185.

11 Kim DK, Nishida H, An SY, Shetty AK, Bartosh TJ, Prockop DJ. Chromatographically isolated CD63+CD81+ extracellular vesicles from mesenchymal stromal cells rescue cognitive impairments after TBI. Proc Natl Acad Sci USA 2016; 113: 170-175.

12 Zhang B, Shi Y, Gong A, Pan Z, Shi H, Yang H et al. HucMSC exosomedelivered $14-3-3 \zeta$ orchestrates self-control of the Wnt response via modulation of YAP during cutaneous regeneration. Stem Cells 2016; 34: 2485-2500

13 Tan CY, Lai RC, Wong W, Dan YY, Lim SK, Ho HK. Mesenchymal stem cell-derived exosomes promote hepatic regeneration in drug-induced liver injury models. Stem Cell Res Ther 2014; 5: 76.

14 Li T, Yan Y, Wang B, Qian H, Zhang X, Shen L et al. Exosomes derived from human umbilical cord mesenchymal stem cells alleviate liver fibrosis. Stem Cells Dev 2013; 22: 845-854.

15 Hyun J, Wang S, Kim J, Kim GJ, Jung Y. MicroRNA125b-mediated Hedgehog signaling influences liver regeneration by chorionic platederived mesenchymal stem cells. Sci Rep 2015; 5: 14135.

16 Ko SF, Yip HK, Zhen YY, Lee CC, Lee CC, Huang CC et al. Adipose-derived mesenchymal stem cell exosomes suppress hepatocellular carcinoma growth in a rat model: apparent diffusion coefficient, natural killer T-cell responses, and histopathological features. Stem Cells Int 2015; 2015 853506.

17 Katsuda T, Kosaka N, Takeshita F, Ochiya T. The therapeutic potential of mesenchymal stem cell-derived extracellular vesicles. Proteomics 2013; 13: 1637-1653.

18 Hyenne V, Apaydin A, Rodriguez D, Spiegelhalter C, Hoff-Yoessle S, Diem $\mathrm{M}$ et al. RAL-1 controls multivesicular body biogenesis and exosome secretion. J Cell Biol 2015; 211: 27-37.

19 Peterson MF, Otoc N, Sethi JK, Gupta A, Antes TJ. Integrated systems for exosome investigation. Methods 2015; 87: 31-45.

20 de Gassart A, Geminard C, Fevrier B, Raposo G, Vidal M. Lipid raft-associated protein sorting in exosomes. Blood 2003; 102: 4336-4344.

21 Braicu C, Tomuleasa C, Monroig P, Cucuianu A, Berindan-Neagoe I, Calin GA. Exosomes as divine messengers: are they the Hermes of modern molecular oncology? Cell Death Differ 2015; 22: 34-45.

22 Schey KL, Luther JM, Rose KL. Proteomics characterization of exosome cargo. Methods 2015; 87: 75-82.

23 Escola JM, Kleijmeer MJ, Stoorvogel W, Griffith JM, Yoshie O, Geuze HJ. Selective enrichment of tetraspan proteins on the internal vesicles of mul-tivesicular endosomes and on exosomes secreted by human B-lymphocytes. J Biol Chem 1998; 273: 20121-20127.

24 Zitvogel L, Regnault A, Lozier A, Wolfers J, Flament C, Tenza D et al. Eradicationof established murine tumors using a novel cell-free vaccine: dendritic cell-derived exosomes. Nat Med 1998; 4: 594-600.

25 Pitt JM, Charrier M, Viaud S, Andre F, Besse B, Chaput N et al. Dendritic cell-derived exosomes as immunotherapies in the fight against cancer. J Immunol 2014; 193: 1006-1011.

26 Anderson MR, Kashanchi F, Jacobson S. Exosomes in viral disease. Neurotherapeutics 2016; 13: 535-546.
27 Schorey JS, Harding CV. Extracellular vesicles and infectious diseases: new complexity to an old story. J Clin Invest 2016; 126: 1181-1189.

28 Fevrier B, Vilette D, Archer F, Loew D, Faigle W, Vidal M et al. Cells release pri-ons in association with exosomes. Proc Natl Acad Sci USA 2004; 101: 9683-9688.

29 Madison MN, Okeoma CM. Exosomes: implications in HIV-1 pathogenesis. Viruses 2015; 7: 4093-4118.

30 Wurdinger T, Gatson NN, Balaj L, Kaur B, Breakefield XO, Pegtel DM. Extracellular vesicles and their convergence with viral pathways. Adv Virol 2012; 2012: 767694.

31 Temme S, Eis-Hübinger AM, McLellan AD, Koch N. The herpes simplex virus-1 encoded glycoprotein B diverts HLA-DR into the exosome pathway. J Immunol 2010; 184: 236-243.

32 Masciopinto F, Giovani C, Campagnoli S, Galli-Stampino L, Colombatto P, Brunetto $\mathrm{M}$ et al. Association of hepatitis $\mathrm{C}$ virus envelope proteins with exosomes. Eur J Immunol 2004; 34: 2834-2842.

33 Hosseini HM, Fooladi AA, Nourani MR, Ghanezadeh F. The role of exosomes in infectious diseases. Inflamm Allergy Drug Targets 2013; 12 : 29-37.

34 Bhatnagar S, Schorey JS. Exosomes released from infected macrophages contain Mycobacterium avium glycopeptidolipids and are proinflammatory. J Biol Chem 2007; 282: 25779-25789.

35 Xiao D, Barry S, Kmetz D, Egger M, Pan J, Rai SN et al. Melanoma cell-derived exosomes promote epithelial-mesenchymal transition in primary melanocytes through paracrine/autocrine signaling in the tumor microenvironment. Cancer Lett 2016; 376: 318-327.

36 Kucharzewska P, Christianson HC, Welch JE, Svensson KJ, Fredlund E, Ringnér $\mathrm{M}$ et al. Exosomes reflect the hypoxic status of glioma cells and mediate hypoxia-dependent activation of vascular cells during tumor development. Proc Natl Acad Sci USA 2013; 110: 7312-7317.

37 Valenti R, Huber V, Filipazzi P, Pilla L, Sovena G, Villa A et al. Human tumor-released microvesicles promote the differentiation of myeloid cells with transforming growth factor-mediated suppressive activity on T Iympho-cytes. Cancer Res 2006; 66: 9290-9298.

38 Zhang L, Zhang S, Yao J, Lowery FJ, Zhang Q, Huang WC et al. Microenvironment-induced PTEN loss by exosomal microRNA primes brain metastasis outgrowth. Nature 2015; 527: 100-104.

39 Stuendl A, Kunadt M, Kruse N, Bartels C, Moebius W, Danzer KM et al. Induction of $\alpha$-synuclein aggregate formation by CSF exosomes from patients with Parkinson's disease and dementia with Lewy bodies. Brain 2016; 139: 481-494.

40 Yuyama K, Sun H, Usuki S, Sakai S, Hanamatsu H, Mioka T et al. A potential function for neuronal exosomes: sequestering intracerebral amyloid- $\beta$ peptide. FEBS Lett 2015; 589: 84-88.

41 Kato T, Miyaki S, Ishitobi H, Nakamura Y, Nakasa T, Lotz MK et al. Exosomes from IL-1beta stimulated synovial fibroblasts induce osteoarthritic changesin articular chondrocytes. Arthritis Res Ther 2014; 16: R163.

42 Sluijter JP, van Rooij E. Exosomal microRNA clusters are important for the therapeutic effect of cardiac progenitor cells. Circ Res 2015; 116: 219-221.

43 Lopez-Verrilli MA, Picou F, Court FA. Schwann cell-derived exosomes enhance axonal regeneration in the peripheral nervous system. Glia 2013; 61: 1795-1806.

44 Haney MJ, Klyachko NL, Zhao Y, Gupta R, Plotnikova EG, He Z et al. Exosomes as drug delivery vehicles for Parkinson's disease therapy. J Control Release 2015; 207: 18-30.

45 Lai RC, Yeo RW, Lim SK. Mesenchymal stem cell exosomes. Semin Cell Dev Biol 2015; 40: 82-88.

46 Lai RC, Tan SS, Teh BJ, Sze SK, Arslan F, de Kleijn DP et al. Proteolytic potential of the MSC exosome proteome: implications for an exosomemediated delivery of therapeutic proteasome. Int J Proteomics 2012; 2012: 971907.

47 Chen TS, Lai RC, Lee MM, Choo AB, Lee CN, Lim SK. Mesenchymal stemcell secretes microparticles enriched in pre-microRNAs. Nucleic Acids Res 2010; 38: 215-224.

48 Maumus M, Jorgensen C, Noël D. Mesenchymal stem cells in regenerative medicine applied to rheumatic diseases: role of secretome and exosomes. Biochimie 2013; 95: 2229-2234.

49 Lai RC, Chen TS, Lim SK. Mesenchymal stem cell exosome: a novel stem cell-based therapy for cardiovascular disease. Regen Med 2011; 6: 481-492. 
50 Lai RC, Yeo RW, Tan KH, Lim SK. Mesenchymal stem cell exosome ameliorates reperfusion injury through proteomic complementation. Regen Med 2013; 8: 197-209.

51 Tian T, Zhu YL, Zhou YY, Liang GF, Wang YY, Hu FH et al. Exosome uptake through clathrin-mediated endocytosis and macropinocytosis and mediating miR-21 delivery. J Biol Chem 2014; 289: 22258-22267.

52 Vickers KC, Remaley AT. Lipid-based carriers of microRNAs and intercellular communication. Curr Opin Lipidol 2012; 23: 91-97.

53 Parolini I, Federici C, Raggi C, Lugini L, Palleschi S, De Milito A et al. Microenvironmental $\mathrm{pH}$ is a key factor for exosome traffic in tumor cells. J Biol Chem 2009; 284: 34211-34222.

54 Rotstein OD, Nasmith PE, Grinstein S. The bacteroides by-product succinicacid inhibits neutrophil respiratory burst by reducing intracellular pH. Infect Immun 1987; 55: 864-870.

55 Marote A, Teixeira FG, Mendes-Pinheiro B, Salgado AJ. MSCs-derived exosomes cell-secreted nanovesicles with regenerative potential. Front Pharmacol 2016; 7: 231.

56 Konala VB, Mamidi MK, Bhonde R, Das AK, Pochampally R, Pal R. The current landscape of the mesenchymal stromal cell secretome: a new paradigm for cell-free regeneration. Cytotherapy 2016; 18: 13-24.

57 Lou G, Song X, Yang F, Wu S, Wang J, Chen Z et al. Exosomes derived from miR-122-modified adipose tissue-derived MSCs increase chemosensitivity of hepatocellular carcinoma. J Hematol Oncol 2015; 8: 122.

58 Fiore EJ, Mazzolini G, Aquino JB. Mesenchymal stem/stromal cells in liver fibrosis: recent findings, old/new caveats and future perspectives. Stem Cell Rev 2015; 11: 586-597.

59 Huang B, Cheng X, Wang H, Huang W, la Ga HuZ, Wang D et al. Mesenchymal stem cells and their secreted molecules predominantly ameliorate fulminant hepatic failure and chronic liver fibrosis in mice respectively. J Trans/ Med 2016; 14: 45.

60 Li J, Ghazwani M, Zhang Y, Lu J, Li J, Fan J et al. miR-122 regulates collagen production via targeting hepatic stellate cells and suppressing P4HA1 expression. J Hepatol 2013; 58: 522-528.

61 Yang JJ, Liu LP, Tao H, Hu W, Shi P, Deng ZY et al. MeCP2 silencing of LncRNA H19 controls hepatic stellate cell proliferation by targeting IGF1R. Toxicology 2016; 359-360: 39-46.

62 Zhou Y, Xu H, Xu W, Wang B, Wu H, Tao Y et al. Exosomes released by human umbilical cord mesenchymal stem cells protect against cisplatininduced renal oxidative stress and apoptosis in vivo and in vitro. Stem Cell Res Ther 2013; 4: 34.

63 Bruno S, Grange C, Deregibus MC, Calogero RA, Saviozzi S, Collino F et al. Mesenchymal stem cell-derived microvesicles protect against acute tubular injury. J Am Soc Nephrol 2009; 20: 1053-1067.

64 Zou X, Zhang G, Cheng Z, Yin D, Du T, Ju G et al. Microvesicles derived from human Wharton's Jelly mesenchymal stromal cells ameliorate renal ischemia-reperfusion injury in rats by suppressing CX3CL1. Stem Cell Res Ther 2014; 5: 40.

65 He J, Wang Y, Sun S, Yu M, Wang C, Pei X et al. Bone marrow stem cellsderived microvesicles protect against renal injury in the mouse remnant kidney model. Nephrology 2012; 17: 493-500.

66 Reis LA, Borges FT, Simoes MJ, Borges AA, Sinigaglia-Coimbra R, Schor N. Bone marrow-derived mesenchymal stem cells repaired but did not prevent gentamicin-induced acute kidney injury through paracrine effects in rats. PLOS ONE 2012; 7: e44092.

67 Zhang $\mathrm{H}$, Xiang M, Meng D, Sun N, Chen S. Inhibition of myocardial ischemia/reperfusion injury by exosomes secreted from mesenchymal stem cells. Stem Cells Int 2016; 2016: 4328362.

68 Lai RC, Arslan F, Lee MM, Sze NS, Choo A, Chen TS et al. Exosome secreted by MSC reduces myocardial ischemia/reperfusion injury. Stem Cell Res 2010; 4: 214-222.

69 Huang L, Ma W, Ma Y, Feng D, Chen H, Cai B. Exosomes in mesenchymal stem cells, a new therapeutic strategy for cardiovascular diseases? Int J Biol Sci 2015; 11: 238-245.

70 Teng X, Chen L, Chen W, Yang J, Yang Z, Shen Z. Mesenchymal stem cellderived exosomes improve the microenvironment of infarcted myocardium contributing to angiogenesis and anti-inflammation. Cell Physiol Biochem 2015; 37: 2415-2424.

71 Zhang Y, Cai W, Huang Q, Gu Y, Shi Y, Huang J et al. Mesenchymal stem cells alleviate bacteria-induced liver injury in mice by inducing regulatory dendritic cells. Hepatology 2014; 59: 671-682.

72 Quintanilha LF, Takami T, Hirose Y, Fujisawa K, Murata Y, Yamamoto N et al. Canine mesenchymal stem cells show antioxidant properties against thioacetamide-induced liver injury in vitro and in vivo. Hepatol Res 2014; 44: E206-E217.
73 Fu J, Zhang $\mathrm{H}$, Zhuang $\mathrm{Y}$, Liu H, Shi Q, Li D et al. The role of $\mathrm{N}$-acetyltransferase 8 in mesenchymal stem cell-based therapy for liver ischemia/reperfusion injury in rats. PLOS ONE 2014; 9: e103355.

74 Zhang J, Zhou S, Zhou Y, Feng F, Wang Q, Zhu X et al. Hepatocyte growth factor gene-modified adipose-derived mesenchymal stem cells ameliorate radiation induced liver damage in a rat model. PLOS ONE 2014; 9: e114670.

75 van Poll D, Parekkadan B, Cho CH, Berthiaume F, Nahmias Y, Tilles AW et al. Mesenchymal stem cell-derived molecules directly modulate hepatocellular death and regeneration in vitro and in vivo. Hepatology 2008; 47: 1634-1643.

76 Chen YX, Zeng ZC, Sun J, Zeng HY, Huang Y, Zhang ZY. Mesenchymal stem cell-conditioned medium prevents radiation-induced liver injury by inhibiting inflammation and protecting sinusoidal endothelial cells. J Radiat Res 2015; 56: 700-708.

77 Bergfeld SA, DeClerck YA. Bone marrow-derived mesenchymal stem cells and the tumor microenvironment. Cancer Metastasis Rev 2010; 29: 249-261.

78 Barcellos-de-Souza P, Comito G, Pons-Segura C, Taddei ML, Gori V, Becherucci $V$ et al. Mesenchymal stem cells are recruited and activated into carcinoma-associated fibroblasts by prostate cancer microenvironment-derived TGF- $\beta 1$. Stem Cells 2016; 34: 2536-2547.

79 Takahara K, Ii M, Inamoto T, Nakagawa T, Ibuki N, Yoshikawa Y et al. microRNA-145 mediates the inhibitory effect of adipose tissue-derived stromal cells on prostate cancer. Stem Cells Dev 2016; 25: 1290-1298.

80 Zhou HS, Su XF, Fu XL, Wu GZ, Luo KL, Fang Z et al. Mesenchymal stem cells promote pancreatic adenocarcinoma cells invasion by transforming growth factor- $\beta 1$ induced epithelial-mesenchymal transition. Oncotarget 2016; 7: 41294-41305.

81 De Boeck A, Pauwels P, Hensen K, Rummens JL, Westbroek W, Hendrix A et al. Bone marrow-derived mesenchymal stem cells promote colorectal cancer progression through paracrine neuregulin 1/HER3 signalling. Gut 2013; 62: 550-560.

82 Norozi F, Ahmadzadeh A, Shahrabi S, Vosoughi T, Saki N. Mesenchymal stem cells as a double-edged sword in suppression or progression of solid tumor cells. Tumour Biol 2016; 37: 11679-11689.

83 Webber J, Yeung V, Clayton A. Extracellular vesicles as modulators of the cancer microenvironment. Semin Cell Dev Biol 2015; 40: 27-34.

84 Roccaro AM, Sacco A, Maiso P, Azab AK, Tai YT, Reagan M et al. BM mesenchymal stromal cell-derived exosomes facilitate multiple myeloma progression. J Clin Invest 2013; 123: 1542-1555.

85 Du T, Ju G, Wu S, Cheng Z, Cheng J, Zou X et al. Microvesicles derived from human Wharton's jelly mesenchymal stem cells promote human renal cancer cell growth and aggressiveness through induction of hepatocyte growth factor. PLOS ONE 2014; 9: e96836.

86 Zhu W, Huang L, Li Y, Zhang X, Gu J, Yan Y et al. Exosomes derived from human bone marrow mesenchymal stem cells promote tumor growth in vivo. Cancer Lett 2012; 315: 28-37.

87 Shi S, Zhang Q, Xia Y, You B, Shan Y, Bao L et al. Mesenchymal stem cell-derived exosomes facilitate nasopharyngeal carcinoma progression. Am J Cancer Res 2016; 6: 459-472.

88 Bruno S, Collino F, Deregibus MC, Grange C, Tetta C, Camussi G. Microvesicles derived from human bone marrow mesenchymal stem cells inhibit tumor growth. Stem Cells Dev 2013; 22: 758-771.

89 Lee JK, Park SR, Jung BK, Jeon YK, Lee YS, Kim MK et al. Exosomes derived from mesenchymal stem cells suppress angiogenesis by downregulating VEGF expression in breast cancer cells. PLOS ONE 2013; 8: e84256.

90 Alcayaga-Miranda F, González PL, Lopez-Verrilli A, Varas-Godoy M, Aguila-Díaz C, Contreras L et al. Prostate tumor-induced angiogenesis is blocked by exosomes derived from menstrual stem cells through the inhibition of reactive oxygen species. Oncotarget 2016; 7: 44462-44477.

91 Klopp AH, Gupta A, Spaeth E, Andreeff M, Marini F 3rd. Concise review: dissecting a discrepancy in the literature: do mesenchymal stem cells support or suppress tumor growth? Stem Cells 2011; 29: 11-19.

92 Ji R, Zhang B, Zhang X, Xue J, Yuan X, Yan Y et al. Exosomes derived from human mesenchymal stem cells confer drug resistance in gastric cancer. Cell Cycle 2015; 14: 2473-2483.

93 Munoz JL, Bliss SA, Greco SJ, Ramkissoon SH, Ligon KL, Rameshwar P. Delivery of functional anti-miR-9 by mesenchymal stem cell-derived exosomes to glioblastoma multiforme cells conferred chemosensitivity. Mol Ther Nucleic Acids 2013; 2: e126. 
94 Mehrotra N, Tripathi RM. Short interfering RNA therapeutics: nanocarriers, prospects and limitations. IET Nanobiotechnol 2015; 9: 386-395.

95 Didiot MC, Hall LM, Coles AH, Haraszti RA, Godinho BM, Chase K et al. Exosome-mediated delivery of hydrophobically modified siRNA for Huntingtin mRNA silencing. Mol Ther 2016; 24: 1836-1847.

$96 \mathrm{Ha} \mathrm{D}$, Yang N, Nadithe V. Exosomes as therapeutic drug carriers and delivery vehicles across biological membranes: current perspectives and future challenges. Acta Pharm Sin B 2016; 6: 287-296.

97 Hood JL, San RS, Wickline SA. Exosomes released by melanoma cells prepare sentinel lymph nodes for tumor metastasis. Cancer Res 2011; 71: 3792-3801.

98 Tan A, Rajadas J, Seifalian AM. Exosomes as nano-theranostic delivery platforms for gene therapy. Adv Drug Deliv Rev 2013; 65: 357-367.

99 Yim N, Ryu SW, Choi K, Lee KR, Lee S, Choi H et al. Exosome engineering for efficient intracellular delivery of soluble proteins using optically reversible protein-protein interaction module. Nat Commun 2016; 7 : 12277.

100 Xitong D, Xiaorong Z. Targeted therapeutic delivery using engineered exosomes and its applications in cardiovascular diseases. Gene 2016; 575: 377-384.

101 Lai RC, Yeo RW, Tan KH, Lim SK. Exosomes for drug delivery-a novel application for the mesenchymal stem cell. Biotechnol Adv 2013; 31: 543-551.

102 Li Q, Wijesekera O, Salas SJ, Wang JY, Zhu M, Aprhys C et al. Mesenchymal stem cells from human fat engineered to secrete BMP4 are nononcogenic, suppress brain cancer, and prolong survival. Clin Cancer Res 2014; 20: 2375-2387.

103 Pascucci L, Coccè V, Bonomi A, Ami D, Ceccarelli P, Ciusani E et al. Paclitaxel is incorporated by mesenchymal stromal cells and released in exosomes that inhibit in vitro tumor growth: a new approach for drug delivery. J Control Release 2014; 192: 262-270.
104 Katakowski M, Buller B, Zheng X, Lu Y, Rogers T, Osobamiro O et al. Exosomes from marrow stromal cells expressing miR-146b inhibit glioma growth. Cancer Lett 2013; 335: 201-204.

105 Shimbo K, Miyaki S, Ishitobi H, Kato Y, Kubo T, Shimose S et al. Exosome-formed synthetic microRNA-143 is transferred to osteosarcoma cells and inhibits their migration. Biochem Biophys Res Commun 2014; 445: 381-387.

106 Kang K, Ma R, Cai W, Huang W, Paul C, Liang J et al. Exosomes secreted from CXCR4 overexpressing mesenchymal stem cells promote cardioprotection via Akt signaling pathway following myocardial infarction. Stem Cells Int 2015; 2015: 659890.

107 Fiore EJ, Bayo JM, Garcia MG, Malvicini M, Lloyd R, Piccioni F et al. Mesenchymal stromal cells engineered to produce IGF-I by recombinant adenovirus ameliorate liver fibrosis in mice. Stem Cells Dev 2015; 24: 791-801.

108 Bliss SA, Sinha G, Sandiford OA, Williams LM, Engelberth DJ, Guiro K et al. Mesenchymal stem cell-derived exosomes stimulate cycling quiescence and early breast cancer dormancy in bone marrow. Cancer Res 2016; 76: 5832-5844.

cc)(ㅇ)

This work is licensed under a Creative Commons Attribution-NonCommercial-NoDerivs 4.0 Inter-

national License. The images or other third party material in this article are included in the article's Creative Commons license, unless indicated otherwise in the credit line; if the material is not included under the Creative Commons license, users will need to obtain permission from the license holder to reproduce the material. To view a copy of this license, visit http://creativecommons.org/licenses/by-nc-nd/4.0/ 